\title{
HONEY BEE OPTIMIZATION BASED SINK MOBILITY AWARE HETEROGENEOUS PROTOCOL FOR WIRELESS SENSOR NETWORK
}

\author{
ASHISH KR. LUHACH* ADITYA KHAMPARIA; RAVINDRA SIHAG $\ddagger$ AND RAJ KUMAR §
}

\begin{abstract}
Wireless Sensor Network (WSN) has emerged as one of the most important technologies serving an array of solutions for critical applications such as defense, industrial monitoring and decision purposes. Data routing in WSN is effective or non-effective depending upon the energy saving for nodes while transferring data packets to the sink. Mainly WSN divided into two modes; heterogeneous and homogeneous. Heterogeneous network in WSN mainly focused on the cluster head selection. Sink mobility in the heterogeneous network has still many open research issues, it is observed that it makes the network more energy efficient. The optimization in the network leads to the stability of the network at a much higher level. In this paper, the sink mobility is optimized for WSN using Honey Bee Optimization (HBO) technique by considering the parameters such as energy and distance. The proposed protocol shows significant improvement in the stability period by $33 \%$ by covering 2928 rounds and enhanced network lifetime by 1500 rounds in compared with 2033 and 14084 rounds for iMBEENISH protocol respectively.
\end{abstract}

Key words: Honey Bee Optimization, Wireless Sensor Network, Swarm intelligence, LEACH protocol.

AMS subject classifications. 68M10, 92B20

1. Introduction. Wireless sensor networks (WSN) sometimes also called as wireless sensor and actor networks (WSAN) are spatially distributed autonomous sensors network to monitor physical or environmental conditions such as temperature, sound, pressure, etc. for critical application such as defense and e-healthcare $[2,17,18]$. The modern sensor networks are bi-directional in the data flow, which means the data can be transmitted from nodes to sink and vice versa enabling control of sensor activity $[19,14,7]$.

The properties of WSN are explained below.

- Infrastructure less: WSN has not any fixed infrastructure, which results in low communication overhead and the network has not forced to follow any specific topology for communication.

- Multi-Hoped: WSN comprises of multiple nodes and the different nodes communicate with each other through different routes to reach each other. In this topology, the data packet travels from one node to another node to reach its final destination. In this way, it helps in energy conservation which is one of the most prominent concerns in WSN [10].

- Requirement of Location information in case of Location Base Algorithm: In case of WSN, the location information is required according to the application for which it is being deployed. Generally, the location information is required in case of geographical based routing protocols. For this purpose, Global Positioning System (GPS) is installed in the sensor nodes. From the installed GPS, they find the distance from the other node and data is sent accordingly.

- Network size: WSN randomly deployed in the remote and large area to sense the information where human intervention is not possible. Generally to cover a large area the number of the node is also more and also a number of the nodes depend on the application for it is used.

- Homogeneous Network and Heterogeneous Networks: WSN may be homogeneous or heterogeneous depending upon the application for which it is used. Heterogeneous networks perform energy efficient routing.

This research work focused on a heterogeneous network of WSN as various clustering topology assign different roles for data collection to the different nodes. The cluster head will consume more energy than the rest of the nodes as it performs more function and operation than others. The research conducted for the same recommends, electing the node having more energy as a cluster head [4] and making it feasible to preserving more energy for the network.

\footnotetext{
${ }^{*}$ The PNG University of Technology, Papua New Guinea (ashishluhach@acm.org ).

${ }^{\dagger}$ Lovely Professional University, Punjab, India

${ }^{\ddagger}$ Banasthali University, Rajasthan, India

$\S$ The PNG University of Technology, Papua New Guinea
} 
2. Related Work. Due to advancement in the field, a lot of research conducted to improve the network lifetime for wireless sensors and energy conversion is one of the most important challenges faced by the researcher. The network stability gets achieved with help of embedding homogeneity in existing networks. To enhance the network lifetime and introduce a variety of energy level nodes in network Stable Election Protocol (SEP) [16] framework was introduced. In this protocol, two types of nodes exist i.e. normal and advance one where an advance node has $(1+a)$ times effective energy than normal one. It helps in enhancing the stability of node i.e. increase the gap time interval before the previous node gets expired. The probability of nodes to be cluster heads is shown in equation 2.1:

$$
P= \begin{cases}\frac{P_{o p t}}{1+a m} & \text { for normal nodes; } \\ \frac{\left(p_{o p t}(1+a)\right)}{(1+a m)} & \text { for advance nodes; }\end{cases}
$$

If two level distributions considered then SEP not retrieved results and get failed. Distributed Energy Efficient Clustering (DEEC) [12] proposed ration of nodes average energy obtained with the residual energy left. DEEC also worked on two levels of energy like SEP. Advanced nodes are having $(1+a)$ times energy than normal one. The probabilistic formula for selection of $\mathrm{CH}$ is given in equation 2.2:

$$
P= \begin{cases}\frac{\left(\left(p_{\text {opt }} E_{i}(r)\right)\right.}{((1+a m) E(r))} & \text { for normal nodes; } \\ \frac{\left(p_{\text {opt }}(1+a) E_{i}(r)\right)}{((1+a m) E(r))} & \text { for advance nodes }\end{cases}
$$

where $E_{i}(r)$ is the residual energy of the node $i$ at round $r, E(r)$ is the average energy at round $r$ of the network which is determined a priori before the nodes are deployed in the network. The formation of cluster heads using advance nodes suffered from penalized effect without consideration of energy reservoir. Developed Distributed Energy-Efficient Clustering (DDEEC) [5] decides the selection of available nodes from cluster heads which are working equally with existing nodes with help of threshold limit. Two levels of heterogeneity protocols proposed where, Energy Efficient Heterogeneous Clustering (EEHC) [9] was the first one who introduced three levels of heterogeneity, containing normal nodes, advanced nodes, and super nodes. The EEHC has $10 \%$ performance improvement over protocol Low Energy Adaptive Clustering Hierarchy (LEACH) [6]. Enhanced Developed Distributed Energy-Efficient Clustering (EDDEEC) [15] worked on threshold values which avoid the energyrich nodes penalizing by existing three levels of nodes formation. Balanced Energy Efficient Network Integrated Super Heterogeneous Protocol (BEENISH) [13] introduced ultra level nodes heterogeneity which comprised of four levels with the involvement of normal, super and advanced nodes. There have been various techniques based on HBO for the mobility of sink in homogeneous WSN $[3,11]$. However, for heterogeneous WSN the mobility of sink is still not touched much.

3. Honey Bee Algorithm for sink mobility in Heterogeneous WSN. Honey Bees algorithm performs random search along with the neighborhood search for both functional and combinatorial optimizations. As shown in Figure 3.1, the main aim of this algorithm is to find an optimal solution by the honey bees natural foraging behavior. Here, various parameters are required in general i.e. scout bees $(n)$, selected sites in visited sites $(m)$, stopping criteria, best sites in selected sites $(e)$, initial patch size that includes the size of the network and its neighborhood, bees for selected sites, bees for $(m-e)$ sites [8]. Bees are randomly placed in a space and then the evaluation of bee's fitness is done. Now, the bees with the highest fitnesses are the selected bees and the bees that visit the sites are selected for the neighborhood search. Now for the selected sites, recruit bees and evaluate fitness. Fittest bees from each patch are selected. Remaining bees are randomly assigned in search space and then their fitness is evaluated. The steps are further repeated until the stopping criterion is met. The bees algorithm is used in various applications such as data clustering, pattern recognition in neural networks, engineering.

In a sensor network, the nodes lying near the sink have to forward the data of their own along with the data of the nodes which are far away from the sink, as a consequence of which the nodes nearby the sink got depleted in terms of their energies. This depletion of the energy of the nearby nodes results in the network isolation or in 


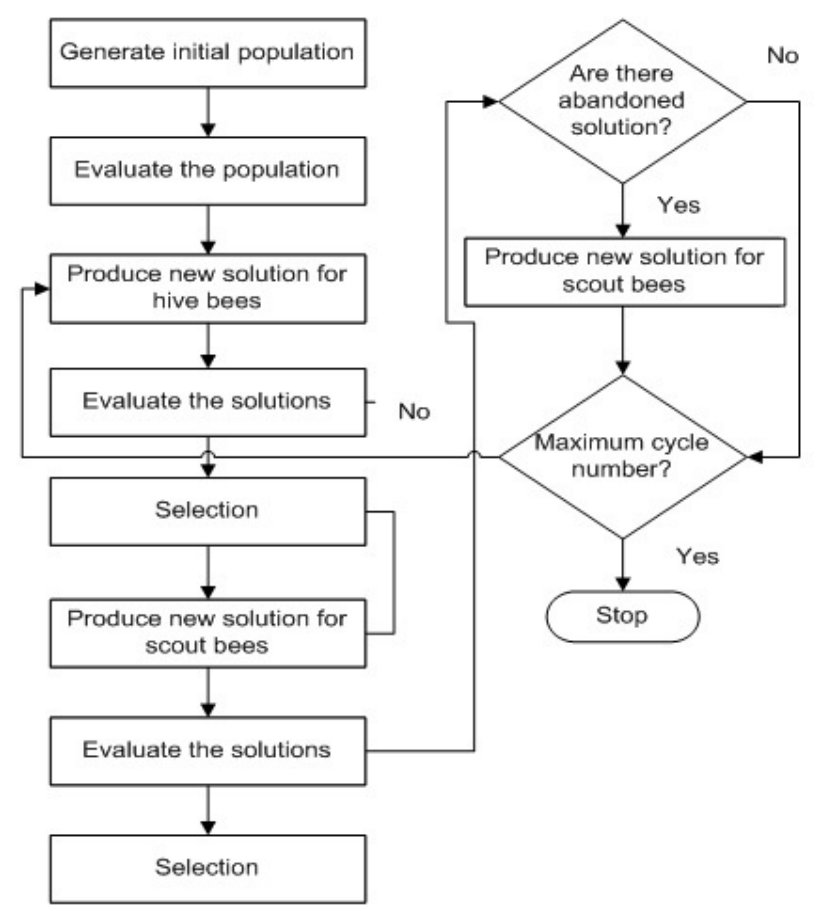

FIG. 3.1. Honey Bees algorithm

other words the HOT SPOT problem. Using sink mobility this problem will be mitigated to a significant level i.e. the energy consumption of the nearby nodes is balanced. There are also some biological protocols which are used to enhance the performance of the sensor network in terms of network lifetime, throughput and quality of service.

4. Proposed Protocol: HBO-iMBEENISH. In this protocol, the research aims to improve mobility aware BEENISH protocol [1]. In this four level of heterogeneity like in BEENISH consisting of normal nodes, advanced nodes, and ultra-nodes. This is the first time any protocol used four levels of energy heterogeneity in the network. $P(i)$ is the probability of the node to become the cluster head and is expressed differently by the different protocols like DEEC, EDEEC, and DDEEC etc. More efficient the probabilistic selection of cluster head more chances of the network having a much better life. As mentioned in the section of problem definition there is penalization of more energy enriched nodes to stay as cluster head which tends to drain their energy much faster when their energy becomes equal to the normal nodes. The concept of a threshold is being applied in the EDDEEC for the three levels of energy heterogeneity but never been done the same in the four levels.

So the new proposed probabilistic equation for the selection of cluster head is given below:

$$
P(i)= \begin{cases}\frac{P o p t * E i}{(1+m(a+m 0(-a+b+m 1(-b+u))) * E a)} & \text { for normnodes if } E(i)>T a b \\ \frac{P o p t * E i *(1+a))}{(1+m(a+m 0(-a+b+m 1(-b+u))) * E a)} & \text { for advnodes if } E(i)>T a b \\ \frac{(P o p t * E i *(1+b))}{(1+m(a+m 0(-a+b+m 1(-b+u))) * E a)} & \text { for supnodes if } E(i)>T a b \\ \frac{(P o p t * E i *(1+u))}{(1+m(a+m 0(-a+b+m 1(-b+u))) * E a)} & \text { for ultnodes if } E(i)>T a b \\ \frac{(c * P o p t * E i *(1+u))}{(1+m(a+m 0(-a+b+m 1(-b+u)) * E a)} & \text { for allnodes if } E(i)>T a b\end{cases}
$$

In above equation the absolute value of Threshold is written as Tab=z*E0 and here $z \in(0,1)$. In our 


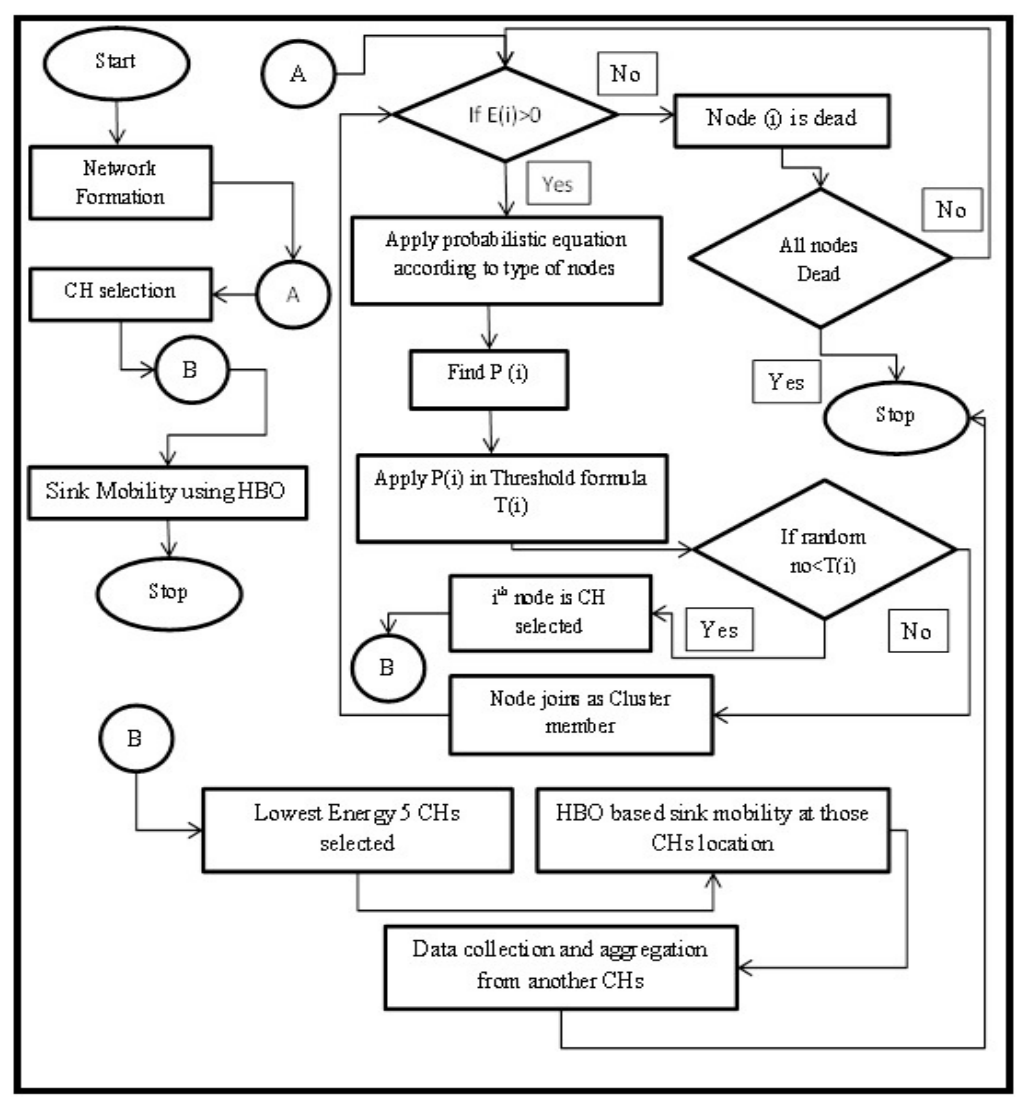

FIG. 4.1. Methodology

proposed scheme we have used $z=0.9$ and $E a$ being the average energy of the network which is given by the expression below:

$$
E a=\frac{E t\left(1-\frac{r}{R}\right)}{n}
$$

Here $r$ is the current round and $\mathrm{R}$ being the maximum number of rounds used in the network. The selection of cluster head is done by the threshold formula which is being used in the BEENISH but with some modification by multiplying it by the terms of residual energy and average energy of network.

$$
T(S i)= \begin{cases}\frac{((P(i) * E(i))}{((1-P(i)(\operatorname{rmod}(1 / P(i))) * E a)} & \text { if } S(i) \in G \\ 0 & \text { otherwise }\end{cases}
$$

Nodes which belong to set $\mathrm{G}$ are eligible for cluster formation or else other nodes get out of cluster if they previously selected. By utilizing value of $P(i)$ using equation 4.3 different normal, ultra and advance nodes selects under cluster head.

The methodology for the proposed work is stated as follows.

i The process for the data collection starts with the network formation as shown in Figure 4.1. In network formation, the network area is defined with a defined number of nodes. The energy values to those nodes are assigned. These energy values are radio values which are fundamentally the same in all wireless routing protocols.

ii Network formation is connected to the connector step $A$. It checks for the energy of every node and applies probabilistic equation according to the types of nodes. If the energy of a node is 0 , a node is 


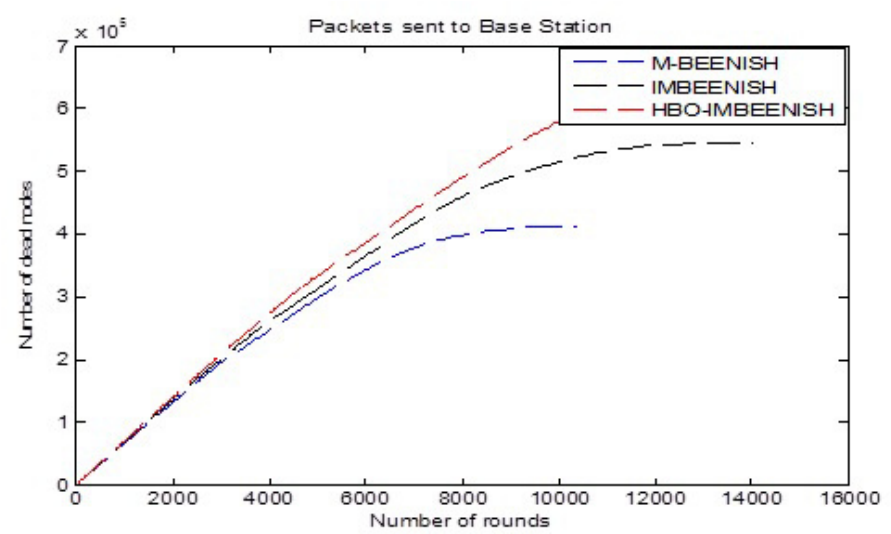

FIG. 5.1. Packets sent to Base Station

said to be dead. A counter is checked for the total number of nodes. If all the nodes are dead, then network stops functioning. If the energy of the node is non zero, then it determines the $P(i)$ value for each node. Then it determines the threshold formula while using the $P(i)$ value.

iii A random number is generated for each node and it is compared with the Threshold value, if the random number is less than the threshold value, it is selected as Cluster Head node. Otherwise, the node is selected as a Cluster Member node.

iv Thereafter, the connection to the $B$ terminal is done. The selection of the lowest energy CHs is done. Honey Bee Optimization is applied to select the $\mathrm{CH}$ and to move the sink towards the selected $\mathrm{CH}$. Then sink collects the data and aggregates from the CHs thereafter the network operation is halted.

5. Results and Discussions. The simulation is performed in MATLAB software. The simulation analysis is being shown between Figure 5.1 - 5.4. It is observed that the HBO-iMBEENISH has outperformed the protocols MBEENISH and iMBEENISH.

Throughput in case of HBO-IMBEENISH has improved significantly as compared to iMBEENISH and MBEENISH as shown in Figure 5.1. It is due to the optimized sink mobility which reduces the number of packet that is dropped while data transmission. The optimized mobility helps in efficient collection of data from the network. The network longevity is observed in Figure 5.1 and Figure 5.3. The stability period is observed to be enhanced by $33 \%$ as compared to iMBEENISH and MBEENISH. It is due to the reduced energy consumption by the proposed approach. The remaining energy of HBO-IMBEENISH covers more number of rounds as compared to the IMBEENISH and MBEENISH as shown in Figure 5.4. It is due to balance in energy consumption in the network by the proposed approach.

6. Conclusion. The growing demand for energy efficient WSN has led to the exponential rise in a communication system by triggering the global academia and industry in the same direction. The mobility in WSN has pushed the horizon of its applications to much further level. It is observed that clustering in WSN has not only brought load balancing in the network but also has distributed the energy consumption evenly in the network. In this paper, applying HBO technique for the sink mobility has not only enhanced stability period but also has improved network lifetime with a much larger amount. The proposed model efficiency gets enhanced to $33 \%$ which covers 2928 rounds and enhance accuracy whereas iMBEENISHpropotcol involves only 2033 rounds. Improvement in network lifetime also moved to 15000 rounds in comparison with the 14084 rounds completed by the iMBEENISH protocol. Total improvement of $6 \%$ gets covered by the proposed protocol. The enhancement in the proposed protocol is accounted to the optimized sink movement in the network and cluster head selection is incorporated with the provision of avoiding the penalization of high energy nodes. The proposed protocol has outperformed the MBEENISH protocol in terms of different performance metrics which are shown in the Figures in the result section. In future work, it is proposed to investigate the performance of the protocol by varying the energy values of nodes. 


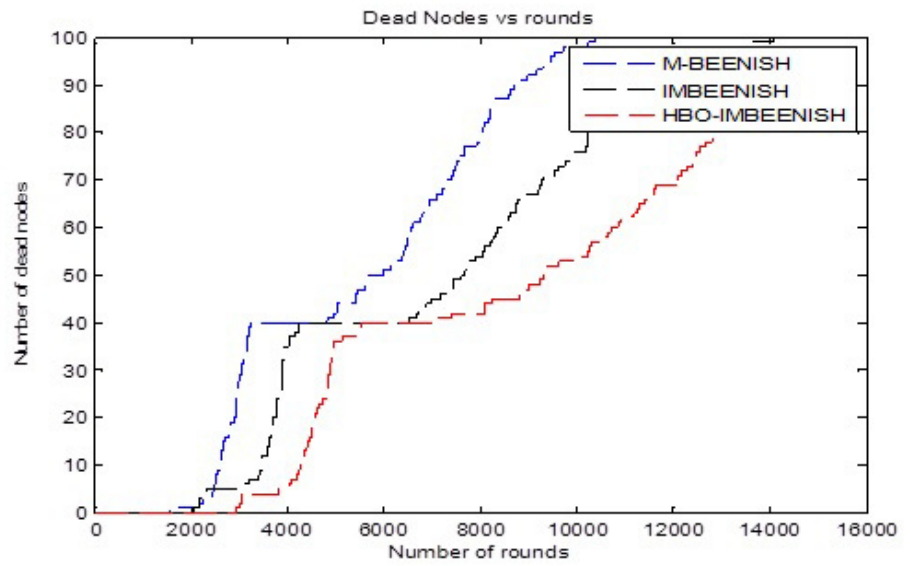

Fig. 5.2. Dead Nodes vs. Rounds

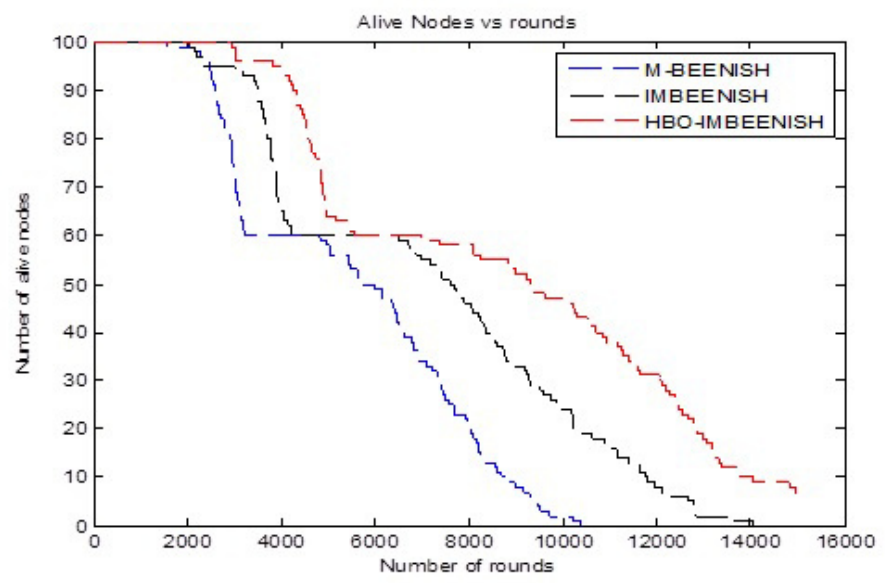

FIG. 5.3. Alive Nodes vs. Rounds

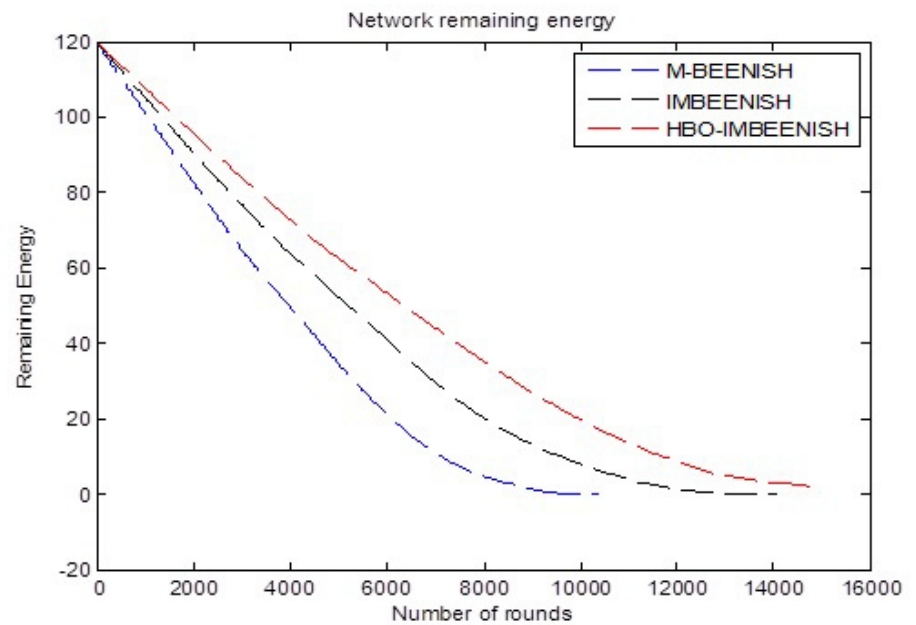

FiG. 5.4. Network Remaining Energy 
Acknowledgments. The authors thanks the anonymous authors whose work largely constitutes the sample file. They also thanks the INFO-TeX mailing list for the valuable indirect assistance they received.

\section{REFERENCES}

[1] M. Akbar, N. Javaid, M. Imran, N. Amjad, M. I. Khan, And M. Guizani, Sink mobility aware energy-efficient network integrated super heterogeneous protocol for wsns, EURASIP Journal on Wireless Communications and Networking, 2016 (2016), p. 66.

[2] I. F. Akyildiz, Weilian Su, Y. Sankarasubramaniam, and E. Cayirci, A survey on sensor networks, IEEE Communications Magazine, 40 (2002), pp. 102-114.

[3] I. BATRA, A. K. LUHACH, AND N. PATHAK, Research and analysis of lightweight cryptographic solutions for internet of things, in Proceedings of the Second International Conference on Information and Communication Technology for Competitive Strategies, ICTCS '16, New York, NY, USA, 2016, ACM, pp. 23:1-23:5.

[4] J. Corchado Rodrguez and A. Abraham, Using heterogeneous wireless sensor networks in a telemonitoring system for healthcare, IEEE Trans. Inform. Technol. Biomed., 14 (2010), pp. 234-240.

[5] B. Elbhiri, R. SaAdane, S. El Fldhi, and D. Aboutajdine, Developed distributed energy-efficient clustering (ddeec) for heterogeneous wireless sensor networks, in 2010 5th International Symposium On I/V Communications and Mobile Network, Sep. 2010, pp. 1-4.

[6] W. R. Heinzelman, A. Chandrakasan, And H. Balakrishnan, Energy-efficient communication protocol for wireless microsensor networks, in Proceedings of the 33rd Annual Hawaii International Conference on System Sciences, Jan 2000, pp. 10 pp. vol.2-.

[7] M. Johnson, M. Healy, P. Van de Ven, M. Hayes, J. Nelson, T. Newe, And E. Lewis, A comparative review of wireless sensor network mote technologies, 11 2009, pp. 1439 - 1442.

[8] D. Karaboga, B. Gorkemli, C. Ozturk, and N. Karaboga, A comprehensive survey: artificial bee colony (abc) algorithm and applications, Artificial Intelligence Review, 42 (2014), pp. 21-57.

[9] D. Kumar, T. C. Aseri, And R. PATEL, Eehc: Energy efficient heterogeneous clustered scheme for wireless sensor networks, Computer Communications, 32 (2009), pp. 662 - 667 .

[10] S. Meguerdichian, F. Koushanfar, G. QU, And M. Potkonjak, Exposure in wireless ad-hoc sensor networks, in Proceedings of the 7th Annual International Conference on Mobile Computing and Networking, MobiCom '01, New York, NY, USA, 2001, ACM, pp. 139-150.

[11] A. Pramanik, A. K. Luhach, I. Batra, And U. Singh, A systematic survey on congestion mechanisms of coap based internet of things, in Advanced Informatics for Computing Research, D. Singh, B. Raman, A. K. Luhach, and P. Lingras, eds., Singapore, 2017, Springer Singapore, pp. 306-317.

[12] L. QING, Q. ZHU, AND M. WANG, Design of a distributed energy-efficient clustering algorithm for heterogeneous wireless sensor networks, Computer Communications, 29 (2006), pp. $2230-2237$.

[13] T. Qureshi, N. Javaid, A. Khan, A. IqBAl, E. AkHtaR, AND M. IshfaQ, Beenish: Balanced energy efficient network integrated super heterogeneous protocol for wireless sensor networks, Procedia Computer Science, 19 (2013), pp. 920 925. The 4th International Conference on Ambient Systems, Networks and Technologies (ANT 2013), the 3rd International Conference on Sustainable Energy Information Technology (SEIT-2013).

[14] K. RMer And F. MATtern, The design space of wireless sensor networks, Wireless Communications, IEEE, 11 (2005), pp. 54 -61 .

[15] P. Saini AND A. K. Sharma, E-deec- enhanced distributed energy efficient clustering scheme for heterogeneous wsn, in 2010 First International Conference On Parallel, Distributed and Grid Computing (PDGC 2010), Oct 2010, pp. 205-210.

[16] G. Smaragdakis, I. Matta, And A. Bestavros, Sep: A stable election protocol for clustered heterogeneous wireless sensor networks, 2004.

[17] S. Sudevalayam and P. Kulkarni, Energy harvesting sensor nodes: Survey and implications, IEEE Communications Surveys and Tutorials, 13 (2011), pp. 443-461.

[18] M. A. M. Vieira, C. N. Coelho, D. C. DA Silva, And J. M. DA Mata, Survey on wireless sensor network devices, in EFTA 2003. 2003 IEEE Conference on Emerging Technologies and Factory Automation. Proceedings (Cat. No.03TH8696), vol. 1, Sep. 2003, pp. 537-544 vol.1.

[19] Y. WANG, Topology Control for Wireless Sensor Networks, Springer US, Boston, MA, 2008, pp. $113-147$.

Edited by: Dharm Singh Jat

Received: Apr 17, 2019

Accepted: Jun 14, 2019 